\title{
PERFIL DEMOGRÁFICO E SÓCIO-ECONÔMICO DO ESTUDANTE DE ENFERMAGEM DA UECE
}

\author{
Maria Salete Bessa Jorge ${ }^{25}$ \\ Maria Luciana Teles Holanda ${ }^{26}$
}

\begin{abstract}
RESUMO: $O$ estudo aborda as principais características dos alunos de enfermagem em relação aos aspectos demográficos, mobilidade geográfica, nivel de instrução, freqüência a outro curso superior, informação sobre a carreira, entre outros itens. Para a coleta de dados, utilizamos questionário com perguntas abertas e fechadas. A análise dos dados pautou-se nas respostas dos alunos, tendo como direcionamento uma visão critica desses dados, os quais foram compilados em forma de tabelas. Os resultados da pesquisa revelaram que a maioria dos alunos de enfermagem é do sexo feminino (88,8\%); $57 \%$ são procedentes de Fortaleza e $60,2 \%$ vivem ainda sob a tutela dos pais. Dos alunos da pesquisa. $18,7 \%$ dos pais têm Curso superior completo e $88,4 \%$ moram em casa alugada.
\end{abstract}

UNITERMOS: Perfil - Estudante de enfermagem

\section{INTRODUÇÃO À QUESTÃO DA PESQUISA}

O estudo tem como objetivo caracterizar o perfil do estudante de enfermagem da Universidade Estadual do Ceará, no que se refere às caracteristicas demográficas, bem como outros aspectos inerentes a esse contexto, envolvendo a origem do aluno e da família. Para tanto,a definição de Perfil profissional deve ser coerente com a ideologia, vocação, politicas sociais e as necessidades de saúde da população que direcionam a organização dos serviços de saúde.

Essa preocupação de conhecer o perfil dos alunos não interessa somente aos sujeitos sociais enfermeiros-docentes da Universidade em questão, mas delineia o pensar de outros pesquisadores envolvidos no processo de ensinoaprendizagem: Nakamae (1989); BOLL (1988) Manzolli \& Monteleone (1977) e Rolin et al (1993), cuja abordagem tem a dimensão de subsidiar o processo ensino-aprendizagem, visando a formação do futuro enfermeiro, adequando-o à realidade das necessidades de saúde da população.

\footnotetext{
25 Enfermeira. Professora da Universidade Estadual do Ceará. Doutoranda em enfermagem da $\mathrm{EERP/EE} \mathrm{/USP}$

26 Aluna bolsista da UECE do $6^{\circ}$ semestre de Enfermagem
} 
Nesse sentido, Nakamae $(1988,1989)$ considera que o processo educacional apresenta adequacidade na medida em que o educador leva em consideração os dados relativos às características dos alunos. Contudo, percebemos que, na maioria das vezes, essas questões deixam de ser valorizadas, dando lugar a outras de menor importância para o ser humano.

Consideramos que esses aspectos assumem relevância no momento da reformulação curricular, tendo como base acurada a análise do papel do enfermeiro na sociedade, de suas funções e do perfil profissional esperado pela sociedade, aspectos dinâmicos que se encontram em constante evolução. No entanto, o perfil do profissional representa o eixo norteador da estrutura dos planos de ensino e da seleção de experiências de aprendizagem, bem como da organização e avaliação do desempenho na prática.

A idéia deste estudo surgiu a partir da inquietação de melhor conhecer os estudantes que optavam pelo Curso de Enfermagem, a fim de compreendê-los no contexto universitário.

\section{METODOLOGIA}

Este estudo baseou-se em dados de uma amostra de estudantes de enfermagem, questionados no mês de Março de 1993 , do $1^{\circ}$ ao $9^{\circ}$ semestres do Curso, colhidos por bolsistas da pesquisa. Tivemos dois momentos de coleta de dados em virtude da Instituição Universitária realizar ingressos desses alunos duas vezes por ano. As amostras, embora coletadas em períodos diversificados, obedeceram aos mesmos critérios básicos. O primeiro consistiu em selecionar os alunos que estavam cursando Enfermagem naquele momento e os que ingressaram através do vestibular no $2^{\circ}$ semestre de 1993. Nesta primeira etapa escolhemos alunos do $1^{\circ}$ ao $9^{\circ}$ semestre do Curso de Enfermagem, para podermos incluir na amostra alunos com vivência universitária e, portanto, mais categorizados para opinarem sobre suas perspectivas profissionais e expressarem seu ponto de vista acerca do mercado de trabalho que os aguarda. O segundo critério foi o aluno presente na ocasião da aplicação do questionário e não o universo dos alunos matriculados. A coleta dos dados foi realizada em dias letivos regulares e em horários intercalados de acordo com a disponibilidade do aluno e do professor.

Os dados amostrados foram coletados através de um questionário contendo 32 perguntas, para o qual se tomou por base a pesquisa de Nakamae (1989). O questionário continha dados, como: idade, sexo, nivel de instrução, freqüência a cursinhos, vestibulares para outros cursos, situação econômica atual do estudante, ocupação remunerada, tipo de auxilio financeiro que recebe, razões da opção pelo curso, entre outros. O instrumento foi distribuído em salas de aula e recolhidos imediatamente após 
seu preenchimento, sem contudo ser limitado o tempo disponivel para as respostas. Procurou-se motivar o aluno, explicando-se-lhe o propósito do trabalho. As dúvidas eram respondidas à medida que surgiam e durante o preenchimento do instrumento.

\section{APRESENTAÇÃO E DISCUSSÃO DOS RESULTADOS}

Os resultados foram analisados à luz dos dados coletados e serão apresentados a seguir:

TABELA 1. Alunos por sexo e semestre que cursaram Enfermagem em 1993 na UECE

\begin{tabular}{|c|c|c|c|c|c|c|c|c|c|c|c|c|c|c|c|c|c|c|c|c|}
\hline \multirow[t]{2}{*}{$\mathrm{SexO}$} & \multicolumn{2}{|c|}{$10 \mathrm{sen}$} & \multicolumn{2}{|c|}{$2 \mathrm{sem}$} & \multicolumn{2}{|c|}{3.5 en } & \multicolumn{2}{|c|}{$4.5 \mathrm{em}$} & \multicolumn{2}{|c|}{$50 \mathrm{sem}$} & \multicolumn{2}{|c|}{$6^{\circ} \mathrm{sem}$} & \multicolumn{2}{|c|}{$7 \mathrm{sem}$} & \multicolumn{2}{|c|}{$88 \mathrm{Sem}$} & \multicolumn{2}{|c|}{$90 \mathrm{em}$} & \multicolumn{2}{|c|}{ Tota } \\
\hline & 10 & 6 & $\mathrm{~N}^{\circ}$ & $\%$ & $\mathrm{~N}^{\circ}$ & 6 & $\mathrm{AO}^{\circ}$ & $\%$ & 10 & 6 & $\mathrm{No}$ & $\%$ & & $1 \%$ & & $\%$ & 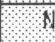 & $\%$ & & 6 \\
\hline Masulino & 11 & 189 & $z$ & 53 & 1 & 58 & 2 & 11 & 4 & 97 & 5 & 172 & 4 & 190 & 2 & 83 & & & 31 & 112 \\
\hline $\mathrm{Ten} \beta \mathrm{O}$ & 47 & 81 & 36 & 94 & 16 & 942 & 26 & 929 & 37 & 903 & 24 & 828 & 11 & 810 & 22 & 91 & 21 & 100 & 246 & 888 \\
\hline Jotal & 38 & 1000 & 38 & 1000 & 17 & 0000 & 28 & 1000 & 41 & 1000 & 29 & 1000 & 21 & 1000 & 24 & 1000 & 21 & 100 & 27 & 1000 \\
\hline
\end{tabular}

A tabela 1, mostra que $246(88,8 \%)$ dos alunos pesquisados são do sexo feminino, tendo atingido no $9^{\circ}$ semestre, $100 \%$ do grupo amostrado.

A origem da profissão de enfermagem é predominantemente feminina. A sociedade colonial brasileira excluia os direitos de cidadania para uma grande maioria da população, composta de negros, pobres, doentes e de mulheres. A familia brasileira era patriarcal e autoritária; mulheres e crianças eram subservientes aos homens privilegiados e dominantes.

C) posigas da mulher is "distintamente inferion, hanto scial come psicologicamente. Como mae "eyposa, o see mundo esti centratizado em vevir o marido, cuidar das rviangas. Quando exisksn decioses importantes a verm lomadas, o julyamento do mavides i dominanle efinal" (Pires, 1989).

Ferreira-Santos (1973) aponta que a profissionalização da mulher através da enfermagem é congruente com os papéis femininos de subordinação aos papéis masculinos, posição esta que coincide com a assimetria vigente na sociedade brasileira contemporânea, que conserva traços da estrutura patriarcal; portanto, a analogia das representações acerca dos papéis da enfermeira e dos papéis maternos reflete-se na concepção da enfermagem como espécie de extensão profissionalizada dos papéis maternos. Essas concepções sobre a enfermagem, que acomodam os papéis profissionais e domésticos da mulher, baseados nas características essenciais para ambos, funcionam paralelamente como elementos negativos na eventual escolha da ocupação por homem. 
Tabela 2. Distribuição dos alunos de Enfermagem por idade- UECE, 1993

\begin{tabular}{|c|c|c|c|c|c|c|c|c|c|c|c|c|c|c|c|c|c|c|c|c|}
\hline \multirow[t]{2}{*}{ lode } & \multicolumn{2}{|c|}{$10 \mathrm{sem}$} & \multicolumn{2}{|c|}{$2^{\circ} \mathrm{sem}$} & \multicolumn{2}{|c|}{$3^{\circ}$ Sem } & \multicolumn{2}{|c|}{$4^{\circ} \mathrm{Sem}$} & \multicolumn{2}{|c|}{$5^{\circ} \mathrm{sem}$} & \multicolumn{2}{|c|}{$6^{\circ}$ Sem } & \multicolumn{2}{|c|}{$7 \% \mathrm{sem}$} & \multicolumn{2}{|c|}{$8^{\circ}$ sem } & \multicolumn{2}{|c|}{$9^{\circ} \mathrm{Sem}$} & \multicolumn{2}{|l|}{ Total } \\
\hline & No & $\%$ & $\mathrm{~N}^{\circ}$ & $\%$ & $N^{0}$ & $\%$ & $N^{\circ}$ & $\%$ & $\mathrm{~N}^{0}$ & $\%$ & $\mathrm{~N}^{0}$ & $\%$ & $\mathrm{~N}$ & $\%$ & $N^{\circ}$ & $\%$ & No & $\%$ & $N^{\circ}$ & $\%$ \\
\hline 620 & 48 & 82.6 & 18 & 47,4 & 2 & 118 & 5 & 178 & 3. & 73 & 1 & 3,5 & & & & & 3 & 14,3 & 80 & 289 \\
\hline 021 & 18 & 12,0 & 9 & 238 & 7 & 41,2 & 13 & 465 & 13 & 31.7 & 14 & 483 & 3 & 14,3 & 2 & 8,3 & 8 & 381 & 76 & 275 \\
\hline 223 & 1 & 18 & 7 & 4 & 3 & 176 & 5 & 178 & 15 & 366 & 7 & $24 / 1$ & 5 & 23,8 & 7 & 292 & 6 & 28,7 & 57 & 20,6 \\
\hline 425 & 1 & 18 & 1 & 26 & 1 & 59 & 1 & 3,6 & 3 & 73 & 2 & 69 & 5 & 23,8 & 4 & 16,7 & 1 & 47 & 19 & 68 \\
\hline 26.27 & 4 & & & & & & 2 & 72 & 2 & 49 & 1 & 3,5 & 1 & 47 & 5 & 208 & & & 12 & 4,3 \\
\hline 28.29 & 1 & 18 & 1 & 2,6 & 2 & 118 & 1 & 3,6 & 1 & 25 & 1 & 3.5 & 3 & 14,3 & 2 & 8,3 & 2 & 9,5 & 13 & 47 \\
\hline$>29$ & & & 1 & 26 & 2 & 118 & 1 & 3.6 & 2 & 49 & 3 & 103 & 4 & 19,1 & 4 & 16,7 & 1 & 4.7 & 19 & 68 \\
\hline$S R$ & & & T) & 26 & & & & & 2 & 4.9 & & & & & 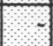 & & & & 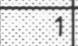 & 0.4 \\
\hline Total & 58 & 100 & 38 & 100 & 17 & 100 & 28 & 100 & 41 & 100 & 29 & 100 & 21 & 100 & 24 & 100 & 21 & 100 & 277 & 100 \\
\hline
\end{tabular}

Observa-se, na tabela 2, que a maior concentração de alunos está na faixa etária menor de 20 anos $(28,9 \%)$. Nakamae \& Costa (1988) apontam que dos alunos das escolas de enfermagem particulares em São Paulo, amostrados em 1988, 55\% encontravam-se nas faixas etárias mais altas e, portanto, com razoável atraso na sua vida escolar. Essa diferença de faixa etária dos alunos que cursam a Universidade Particular, provavelmente acontece devido à problemática social do estudante, que é a dificuldade de ingressar no $1^{\circ} \mathrm{Grau}$ com a idade escolar estabelecida pelo Conselho de Educação (7anos). Com isso, ele perde a oportunidade de concorrer com alunos de "status" sócioeconômico mais elevado, de conviver ainda na mesma fase de adolescência, pois, nesse momento, já está participando com atividades econômıcas necessárias para ajudar no sustento da familia e no auto-sustento. Portanto, a única alternativa é tentar uma vaga na Universidade particular.

Percebe-se que a maioria dos alunos que estão cursando Enfermagem em 1993 na UECE é constituída de jovens, compreendidoss em uma faixa de menos de 20anos a 25anos. Idade considerada por muitos autores como final da adolescência, entre 18 e 20 anos, quando o jovem torna-se legalmente capaz de assumir responsabilidades de trabalho. (COMITÉ DE TÉCNICOS DA ORGANIZAÇÃO MUNDIAL DE SAÚDE, 1975). Schmiedelk (1979) afirmou que é nessa fase que o jovem, utilizando o pensamento forma, tem capacidade intelectual para entender conceitos hipotéticos e abstratos, projetando-os para si mesmos.

Caldas (1991) afirmou que a adolescência não é somente uma fase estanque do desenvolvimento como é preconizado por muitos, mas sim um tempo, uma forma de viver e um modo de ser no mundo, não se podendo, portanto, demarcar idades exatas para situá-las e nem tão pouco um conceito absoluto ou uma definição universal que, categoricamente, exprima o que é ser adolescente. 
Tabela 3. Distribuição dos estudantes da pesquisa do $1^{\circ}$ ao $9^{\circ}$ semestre segundo sua origem. 1993

\begin{tabular}{|c|c|c|c|c|c|c|c|c|c|c|c|c|c|c|c|c|c|c|c|c|}
\hline \multirow[t]{2}{*}{ Ongem } & \multicolumn{2}{|c|}{$1^{\circ} \mathrm{Sem}$} & \multicolumn{2}{|c|}{$2^{\circ}$ Sem } & \multicolumn{2}{|c|}{$3^{\circ} \mathrm{Sem}$} & \multicolumn{2}{|c|}{$4^{\circ}$ Sem } & \multicolumn{2}{|c|}{$5^{\circ}$ Sem } & \multicolumn{2}{|c|}{$6^{\circ}$ Sem } & \multicolumn{2}{|c|}{$7^{\circ}$ Sem } & \multicolumn{2}{|c|}{$8^{\circ} \mathrm{Sem}$} & \multicolumn{2}{|c|}{$9^{\circ} \mathrm{sem}$} & \multicolumn{2}{|c|}{ Totat } \\
\hline & $N^{9}$ & $\%$ & $N^{\circ}$ & $\%$ & $N^{0}$ & $\%$ & $\mathrm{~N}$ & $\%$ & No & $\%$ & $N^{\circ}$ & $\%$ & $N^{\circ}$ & $\%$ & $N^{6}$ & $\%$ & $\mathrm{~N}^{\circ}$ & $\%$ & $N^{\circ}$ & $\%$ \\
\hline Acre & 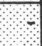 & 1 & 9 & 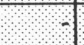 & 1 & & $=$ & & 2 & 4.8 & 2 & 69 & 8 & & 9 & 8 & 8 & & 4 & 15 \\
\hline Alagoas & 3 & $\Rightarrow$ & -1 & 3 & 8 & & 1 & -8 & 1 & 2,5 & 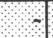 & & 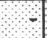 & 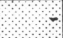 & 1 & 9 & & 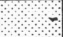 & 1 & 04 \\
\hline $\mathrm{AM}$ & 9 & & 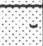 & 3 & 5 & 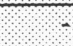 & 9 & - & 9 & 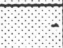 & 8 & 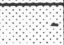 & 8 & 9 & 1 & 42 & 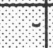 & 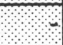 & 1 & 04 \\
\hline Bahia & 1 & 17 & 1 & 26 & 5 & & I & 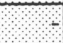 & 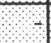 & 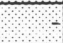 & + & 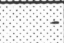 & 1 & 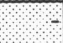 & 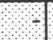 & & 8 & 8 & 2 & 01 \\
\hline Ceara & 49 & 84,5 & 31 & 820 & 16 & 941 & 26 & 930 & 30 & 73,3 & 25 & 86.1 & 19 & 905 & 21 & 875 & 18 & 860 & 235 & 850 \\
\hline $\mathrm{MA}$ & 1 & 17 & 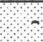 & 3 & 3 & 8 & 9 & & 1 & 25 & 0 & 8 & 8 & 8 & 9 & 8 & $\$$ & 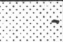 & 2 & 87 \\
\hline Praut & 2 & 35 & 8 & 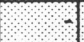 & 1 & 59 & 1 & 3,5 & 1 & 4.8 & 9 & 8 & 8 & 8 & 3 & 8 & 5 & 勧 & 5 & 1.8 \\
\hline Para & $\$$ & & 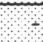 & 3 & 3 & 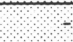 & 3 & 8 & 2 & 4,8 & 8 & 3 & 8 & s & 5 & 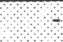 & a & 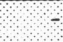 & 2 & $8 \%$ \\
\hline $\mathrm{RN}$ & 3 & & 2 & 50 & 5 & 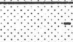 & 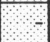 & 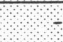 & 1 & 2,5 & 1 & 3,5 & 2 & 95 & 8 & 8 & 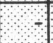 & 8 & 6 & 22 \\
\hline $\mathrm{R}$ & 3 & 5.2 & 1 & 26 & 9 & 3 & 1 & 3,5 & 8 & 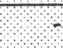 & 1 & 35 & 8 & 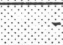 & 1 & 6 & 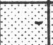 & 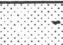 & 6 & 22 \\
\hline $\mathrm{RS}$ & \% & 8 & 1 & 26 & 3 & 3 & 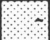 & 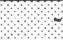 & 1 & 8 & 1 & 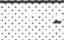 & 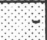 & 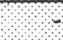 & 3 & 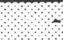 & 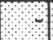 & 8 & 1 & 04 \\
\hline$S P$ & 1 & 17 & 1 & 26 & 3 & 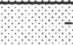 & $=$ & 8 & 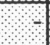 & & 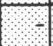 & 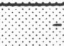 & 8 & 8 & + & 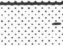 & 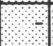 & 8 & 2 & 07 \\
\hline$S R$ & 1 & 17 & 1 & 26 & 3 & 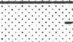 & $=$ & 7 & 2 & 4,8 & - & 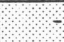 & 7 & 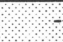 & 2 & 8,3 & 3 & 140 & 9 & 33 \\
\hline Total & 58 & 100 & 38 & 100,0 & 17 & 100,0 & 28 & 100 & 41 & 100 & 29 & 100 & 21 & 100 & 24 & 100 & 21 & 100 & 27 & 100 \\
\hline
\end{tabular}

Legenda: AM- Amazonas; MA - Maranhão; RN - Rio Grande do Norte; RJ Rio de Janeiro; RS-Rio Grande do Sul, SP - São Paulo; SR - Sem referência

Observa-se, na Tabela acima, que 235 (85,0\%) dos alunos amostrados são provenientes do Ceará, enquanto $32(11,5 \%)$ são de outros Estados brasileiros. Esse quantitativo de alunos do Estado do Ceará é elevado em virtude de, sendo eles de origem do referido Estado, não necessitarem deslocar-se com a finalidade de ingressarem em uma Instituição de nível superior, já que tiveram a oportunidade impar.

Tabela 4. Distribuição dos estudantes do $1^{\circ}$ ao $9^{\circ}$ semestre, segundo o local de residência. 1993

\begin{tabular}{|c|c|c|c|c|c|c|c|c|c|c|c|c|c|c|c|c|c|c|c|c|}
\hline \multirow[t]{2}{*}{ Restodencia } & \multicolumn{2}{|c|}{10 Sem } & \multicolumn{2}{|c|}{$2 \mathrm{sem}$} & \multicolumn{2}{|c|}{$3^{6} \mathrm{Sem}$} & \multicolumn{2}{|c|}{$4^{\circ} \mathrm{Sem}$} & \multicolumn{2}{|c|}{$5^{6} \mathrm{cem}$} & \multicolumn{2}{|c|}{$6^{\circ} \mathrm{sem}$} & \multicolumn{2}{|c|}{$70 \mathrm{sem}$} & \multicolumn{2}{|c|}{$80 \mathrm{sem}$} & \multicolumn{2}{|c|}{$90 \mathrm{sem}$} & \multicolumn{2}{|c|}{ Totio } \\
\hline & $N^{0}$ & $\%$ & $N^{0}$ & $\%$ & No & $\%$ & $\mathrm{~N}^{0}$ & 8 & $N^{\circ}$ & $\%$ & $\mathrm{~N}$ & $\%$ & No & $\%$ & No & o & No & 6 & 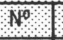 & $\%$ \\
\hline past dos & 52 & 898 & 23 & 605 & 9 & 529 & 19 & 680 & 27 & 66,0 & 14 & 483 & 9 & 42,8 & 11 & 458 & 11 & 524 & 167 & 603 \\
\hline Sozmho & 2 & 34 & 1 & 26 & 1 & 58 & & & 3 & 73 & 2 & 69 & 1 & 48 & 1 & 44 & 1 & 41 & 2 & 4 \\
\hline Conjuglue & 1 & 17 & 4 & 105 & 1 & 58 & 3 & 106 & 3 & 13 & 61 & 202 & 9 & 33 & 4 & 166 & 4 & 19 & 32 & 106 \\
\hline $\mathrm{Sensao}$ & 1 & 17 & 1 & 26 & & & & & & & & & & & & & & & 2 & 07 \\
\hline Parente & 1 & 17 & 3 & 186 & 5 & 29 & 6 & 214 & 6 & 14.6 & 6 & 207 & 3 & 143 & 4 & 16.6 & 4 & 191 & 51 & 184 \\
\hline Republica & 1) & 17 & 1 & 26 & 3 & & 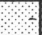 & & & & 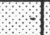 & & 1 & 8 & 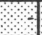 & & 1 & 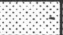 & 2 & 07 \\
\hline $\mathrm{SR}$ & 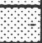 & & 1 & 26 & 1 & 38 & & & 2 & 48 & 1 & 34 & 1 & 48 & 4 & 16.6 & T) & 4 & 11 & 40 \\
\hline Total & 58 & 100 & 38 & 100 & 17 & 100 & 28 & 100 & 41 & 100 & 29 & 100 & 24 & 100 & 24 & 100 & 21 & 100 & 27 & 100 \\
\hline
\end{tabular}


Pela amostra estudada, evidencia-se que os estudantes universitários ainda estão vivendo sob a tutela dos pais, 167 (60,3\%), ou com parentes, $51(18,4 \%)$, enquanto $12(4,3 \%)$ desses alunos de enfermagem moram sozinhos e $02(0,7 \%)$ em república. Isso vem corroborar a idéia de que poucos são os alunos que se emanciparam da tutela dos pais, perfazendo um total de $16(5,8 \%)$, que representa uma parcela muito pequena em relação à amostra total. Esses dados indicam a persistência de traços culturais paternalistas, de proteção absoluta aos filhos, tirando a iniciativa deles próprios tomarem suas próprias decisões e decidirem sobre os destinos de sua vida. Esse quadro revela a supervalorização dada ao curso superior, de que só através dele é possivel emancipar-se, ter projeção social e estabilidade profissional,

Tabela 5. Distribuição dos estudantes do $1^{\circ}$ ao $9^{\circ}$ semestre de Enfermagem quanto à situação de moradia dos pais e alunos. 1993

\begin{tabular}{|c|c|c|c|c|c|c|c|c|c|c|c|c|c|c|c|c|c|c|c|c|}
\hline \multirow[t]{2}{*}{ Resudon 0 a } & \multicolumn{2}{|c|}{18 sen } & \multicolumn{2}{|c|}{$z^{\circ} \mathrm{sen}$} & \multicolumn{2}{|c|}{$0 \mathrm{sen}$} & \multicolumn{2}{|c|}{$40 \mathrm{sen}$} & \multicolumn{2}{|c|}{$5 \% \mathrm{sem}$} & \multicolumn{2}{|c|}{60} & \multicolumn{2}{|c|}{$9 \mathrm{sem}$} & \multicolumn{2}{|c|}{$6 \mathrm{sen}$} & \multicolumn{2}{|c|}{$9 \mathrm{Sen}$} & \multicolumn{2}{|l|}{ otal } \\
\hline & $\mathrm{N}^{\circ}$ & 8 & $\mathrm{~N} 6$ & $\%$ & $6 \%$ & $\%$ & 19 & $\%$ & 10 & $\%$ & $\mathrm{~N}^{6}$ & $\%$ & $\kappa^{6}$ & \% & $1^{\circ}$ & $\%$ & 6 & 6 & 6 & $\%$ \\
\hline Propha & 49 & 845 & 36 & 94 & 16 & 941 & 24 & 85 & 33 & 805 & 25 & 862 & 8 & 100 & 22 & 916 & 1 & 809 & 445 & $88 \%$ \\
\hline Alugada & 9 & 15, & 2 & 53 & 1 & 59 & 4 & 143 & 8 & 195 & 4 & 138 & & & 2 & 84 & 4 & 91 & 29 & 101 \\
\hline $\mathrm{SH}$ & 0 & 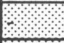 & 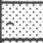 & & 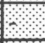 & 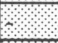 & 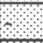 & \% & & & 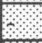 & 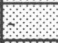 & \% & 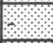 & 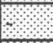 & 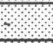 & 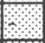 & 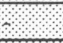 & 9 & 12 \\
\hline Sota & 58 & 100 & 38 & 100 & 17 & 100 & 28 & 100 & 44 & 100 & 29 & 100 & 21 & 100 & 24 & 100 & 21 & 100 & 27 & 180 \\
\hline
\end{tabular}

Legenda: SR - sem referência

Pelos dados amostrados na Tabela 5, percebe-se que a maioria dos alunos reside em casa própria, $245(88,4 \%)$ e uma pequena parcela reside em casa alugada, 29 (10,4\%). Tomando tais infomações como um dos indices de "status" sócio-econômico, identifica-se que os estudantes estão em uma classe privilegiada, pois a maioria do povo brasileiro ainda não possui um teto para morar.

Tabela 6. Alunos que estudaram em cursinho para ingressar na Universidade - 1993

\begin{tabular}{|c|c|c|c|c|c|c|c|c|c|c|c|c|c|c|c|c|c|c|c|c|}
\hline \multirow[t]{2}{*}{ Qursolo } & \multicolumn{2}{|c|}{ Sen } & \multicolumn{2}{|c|}{$2^{6} \mathrm{Sem}$} & \multicolumn{2}{|c|}{$3 \mathrm{Sen}$} & \multicolumn{2}{|c|}{$4 \mathrm{Sen}$} & \multicolumn{2}{|c|}{$5 \mathrm{sen}$} & \multicolumn{2}{|c|}{$64 \mathrm{Sen}$} & \multicolumn{2}{|c|}{ S Sen } & \multicolumn{2}{|c|}{865} & \multicolumn{2}{|c|}{68 Ser } & \multicolumn{2}{|c|}{ 1otal } \\
\hline & $N$ & $\%$ & m & $\%$ & $\mathrm{No}$ & 6 & A & $\%$ & 10 & $\%$ & Ho & 8 & $N o$ & $\%$ & Ho & 6 & No & $\%$ & \% & $\%$ \\
\hline $\mathrm{sin}$ & 30 & 317 & 16 & 647 & 14 & 642 & 15 & 535 & 1 & 470 & 13 & 448 & 9 & 418 & 10 & 416 & 8 & 800 & 129 & 465 \\
\hline N & 26 & 446 & 6 & 353 & 6 & 35 & 12 & 48 & 24 & 590 & 16 & 45 & 12 & $58 z$ & 10 & 416 & 12 & $5>1$ & 134 & 483 \\
\hline $\mathrm{SA}$ & 2 & 35 & & & & & 3 & 3 & & & & & & & 4 & 168 & 1 & 49 & 4 & 5 \\
\hline ood & 58 & 100 & 38 & 100 & 1 & 100 & 28 & 100 & 81 & 100 & 9 & 100 & 21 & 100 & 24 & 100 & 21 & 100 & 27 & 100 \\
\hline
\end{tabular}

A maioria dos alunos amostrados não frequentou cursinho para ingressar na Universidade Estadual do Ceará, 134( 48,3\%). Isso provavelmente decorre de os alunos possuirem um poder aquisitivo melhor, tendo estudado em colégios particulares, ou pelo próprio esforço. Desses dados, ainda pode-se perceber que $129(46,5 \%)$ dos alunos da pesquisa estudaram em cursinho. 
Para Nakamae (1989)7, esses estudantes que buscam os "cursinhos preparatórios, provavelmente o fazem em virtude da maior exigência nos exames vestibulares para ingresso, como também pela possivel deficiência nos cursos de $2^{\circ}$ Grau do País.

Tabela 7. Alunos de enfermagem que prestaram vestibular para outros cursos em outras instituições de Nível Superior. 1993

\begin{tabular}{|c|c|c|c|c|c|c|c|c|c|c|c|c|c|c|c|c|c|c|c|c|}
\hline \multirow[b]{2}{*}{ Vessbularo } & \multicolumn{2}{|c|}{$0 \mathrm{Sen}$} & \multicolumn{2}{|c|}{$8 \mathrm{Sen}$} & \multicolumn{2}{|c|}{$38 \mathrm{sem}$} & \multicolumn{2}{|c|}{$4 \mathrm{Cen}^{2}$} & \multicolumn{2}{|c|}{$5 \mathrm{sen}$} & \multicolumn{2}{|c|}{$6^{\circ} \mathrm{sen}$} & \multicolumn{2}{|c|}{$65 e n$} & \multicolumn{2}{|c|}{8 Sem } & \multicolumn{2}{|c|}{$9 \mathrm{sem}$} & \multicolumn{2}{|c|}{$6+2$} \\
\hline & 10 & $\%$ & $\sqrt{10}$ & 6 & Nit & 6 & 10 & 6 & N & 6 & $\mathrm{No}$ & $\%$ & A\% & 6 & 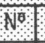 & $\%$ & 9 & $\%$ & $1 \mathrm{~N}$ & $\%$ \\
\hline $\mathrm{sm}$ & 55 & 948 & 32 & 848 & 17 & 100 & 23 & 821 & 17 & 48 & 26 & 896 & 20 & 952 & 81 & 875 & 18 & $86 \%$ & 29 & 826 \\
\hline 10\% & 3 & 8 & 3 & 19 & & & 3 & 101 & 24 & 58 & 3 & 10,4 & 1 & 18 & 3 & 12 & 3 & 13 & 43 & 156 \\
\hline A & & & 3 & 9 & & & 2 & 72 & & & 8 & & & & 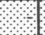 & & 3 & & 3 & 18 \\
\hline ota & 8 & 100 & 38 & 100 & 9 & 100 & 28 & 00 & 4 & 100 & 29 & 100 & 21 & 100 & 24 & 100 & 2 & 100 & ry & 10 \\
\hline
\end{tabular}

Legenda SR-sem referência

A Tabela 7 mostra que 229 (82,6\%) dos alunos de enfermagem amostrados prestaram vestibular para outros cursos. O fato de terem ou não prestado vestibular para outro curso é apontado por Pastore \& Perosa (1971) como "grau de certeza" que os estudantes possuem de sua opção por um determinado ramo. Entre os alunos amostrados, a maioria indicou ter prestado exame para outro curso, e estes dados são também confirmados no estudo de Nakamae (1989).

Tabela 8. Alunos que completaram outro Curso Superior e cursam Enfermagem-1993

\begin{tabular}{|c|c|c|c|c|c|c|c|c|c|c|c|c|c|c|c|c|c|c|c|c|}
\hline Conpletaran & \multicolumn{2}{|c|}{$10 \mathrm{sen}$} & \multicolumn{2}{|c|}{$2 \mathrm{sen}$} & \multicolumn{2}{|c|}{$36 \mathrm{sem}$} & \multicolumn{2}{|c|}{4 Sen } & \multicolumn{2}{|c|}{$5 \mathrm{Sem}$} & \multicolumn{2}{|c|}{$66 \mathrm{sen}$} & \multicolumn{2}{|c|}{$10 \mathrm{sen}$} & \multicolumn{2}{|c|}{$8 \mathrm{Sem}$} & \multicolumn{2}{|c|}{$9 \mathrm{Sem}$} & \multicolumn{2}{|c|}{ 100 } \\
\hline 04 ro Cusso & 10 & $\%$ & 60 & $\%$ & $10 \%$ & $\%$ & NO & $\%$ & 10 & $\%$ & $N^{\circ}$ & $\%$ & $\mathrm{No}$ & $\%$ & 19 & $\%$ & $N^{\circ}$ & \% & $\mathrm{N} \cdot \mathrm{O}$ & $\%$ \\
\hline 6 & & & & & 2 & 18 & & & 3 & 14 & 2 & 69 & & & 4 & 56 & 4 & 1913 & 13 & 4 \\
\hline Và & 56 & 26.5 & 38 & 100 & 15 & 88 & 88 & 100 & 38 & 926 & 2 & 931 & 21 & 100 & 20 & 83,3 & 14 & 809 & 260 & 938 \\
\hline $\mathrm{B}$ & 2 & 35 & & & & & & & & & & & & & & & & & 2 & 08 \\
\hline cotal & 58 & 10 & 38 & 100 & 11 & 100 & 28 & 100 & 41 & 100 & 29 & 100 & 21 & 100 & 24 & 100 & 21 & 100 & 27 & 100 \\
\hline
\end{tabular}

Os dados da Tabela 8 mostram que 260 (93,8\%) dos estudantes de enfermagem não têm outro curso superior, enquanto uma pequena minoria prestou vestibular para outro curso. Entretanto, Nakamae (1989) refere que o aluno ao ser inquirido sobre a escolha pela enfermagem, aponta a inclinação pela profissão, e que, entre os reprovados em outros cursos, ao tentarem ingressar, através do vestibular, se percebe que é expressiva a procura por ramos socialmente muito valorizados, como Medicina e Odontologia, bastante procurados e que põem dificuldades maiores para a aprovação de grande número de vestibulandos. Estes dados também são identificados nesta pesquisa, os quais serão divulgados posteriormente. 
Tabela 9. Distribuição dos estudantes do $1^{\circ}$ ao $9^{\circ}$ semestre, segundo o local de residência. 1993

\begin{tabular}{|c|c|c|c|c|c|c|c|c|c|c|c|c|c|c|c|c|c|c|c|c|}
\hline \multirow[t]{2}{*}{ hesdéncia } & \multicolumn{2}{|c|}{$0 \mathrm{sen}$} & \multicolumn{2}{|c|}{$2 \mathrm{sen}$} & \multicolumn{2}{|c|}{$30 \mathrm{sem}$} & \multicolumn{2}{|c|}{$4 . \mathrm{Sen}$} & \multicolumn{2}{|c|}{$S^{0} \mathrm{Sen}$} & \multicolumn{2}{|c|}{$608 \mathrm{em}$} & \multicolumn{2}{|c|}{6 sen } & \multicolumn{2}{|c|}{$8.5 e n$} & \multicolumn{2}{|c|}{$9 \mathrm{sen}$} & \multicolumn{2}{|c|}{ Tota } \\
\hline & $\mathrm{N}^{\circ}$ & $\%$ & $\mathrm{No}$ & \% & No & $\%$ & $N 1$ & $\%$ & $\mathrm{~N}$ & $\%$ & $\mathrm{~N}^{\circ}$ & $\%$ & $\mathrm{Nol}$ & \% & लm & 6 & No & $\%$ & $N^{\circ}$ & $\%$ \\
\hline Casa dos pais & 52 & 898 & 23 & 605 & 9 & 529 & 19 & 680 & 27 & 660 & 14 & 483 & 9 & 428 & 11 & 45 & 11 & 524 & 167 & 60,3 \\
\hline Sozbhoo & 2 & 34 & 1 & 26 & 1 & 58 & & & 3 & 13 & 1 & 69 & 1 & 48 & 1 & 44 & 1 & 4 & 12 & 43 \\
\hline Congugue & 1 & 17 & 4 & 105 & I & 58 & 3 & 10.6 & 3 & 23 & 6 & 200 & 7 & 33 & 4 & 16.6 & 4 & 191 & 32 & 16 \\
\hline $\mathrm{Pens}+$ & 1 & 17 & 1 & 26 & & & & & & & & & & & & & & & 2 & 0 \\
\hline Parente & 3 & 17 & 7 & 186 & 3 & 297 & 6 & 214 & 6 & 146 & 6 & 207 & 3. & 143 & 4 & 166 & 4 & 191 & 5 & 184 \\
\hline Cepubios & 4 & 18 & 1 & 26 & : & & & & & & & & 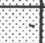 & & & & & & 2 & $0 \%$ \\
\hline 5 & 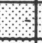 & 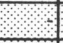 & 1 & 26 & 1 & 58 & 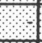 & & 2 & 4,8 & 1 & 34 & 1 & 4,8 & 4 & 16.6 & 1 & 42 & 11 & 40 \\
\hline 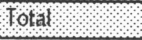 & 58 & 100 & 38 & 100 & 17 & 100 & 28 & 100 & 41 & 100 & 29 & 100 & 21 & 100 & 24 & 100 & 21 & 100 & 271 & 100 \\
\hline
\end{tabular}

Percebe-se que 191 (69\%) dos alunos amostrados na Tabela 9 apontam, como razões para escolha da profissão, inclinação pela profissão; $17(6,1 \%$ ) indicam influência de parentes, enquanto $15(5,4 \%)$ procuraram o teste vocacional. Ao analisarmos esses dados, observa-se que a escolha por indicação de parentes nos deixa uma interrogação: Como será que esse profissional vai enfrentar o assistir ao paciente, as dificuldades da profissão, se a escolha desta foi realizada de acordo com o desejo de parentes?

Uma pequena percentagem de alunos, $53(19,1 \%)$, deixou de responder qual o motivo que os levou a fazer Enfermagem. Isso nos induz ao seguinte questionamento: Será que o aluno sente-se confuso, inseguro?

Tabela 10: Expectativas dos alunos de enfermagem a respeito de suas oportunidades de emprego. 1993

\begin{tabular}{|c|c|c|c|c|c|c|c|c|c|c|c|c|c|c|c|c|c|c|c|c|}
\hline \multirow{2}{*}{ Expeo } & \multicolumn{2}{|c|}{$8 \mathrm{sem}$} & \multicolumn{2}{|c|}{$2^{\circ} \mathrm{em}$} & \multicolumn{2}{|c|}{$3 \mathrm{sen}$} & \multicolumn{2}{|c|}{$40 \mathrm{sen}$} & \multicolumn{2}{|c|}{$58 \mathrm{sem}$} & \multicolumn{2}{|c|}{$6^{\circ} \mathrm{Sem}$} & \multicolumn{2}{|c|}{$7 \mathrm{Sen}$} & \multicolumn{2}{|c|}{$80 \mathrm{sem}$} & \multicolumn{2}{|c|}{$90 \mathrm{Sen}$} & \multicolumn{2}{|c|}{ Total } \\
\hline & $\mathrm{NO}$ & $\%$ & $N^{\circ}$ & $\%$ & $N$ & $\%$ & $\mathrm{No}$ & $\%$ & $\mathrm{~N}^{0}$ & $\%$ & No & $\%$ & No & $\%$ & $N^{\circ}$ & $\%$ & No & $\%$ & $\mathrm{NO}^{\circ}$ & $\%$ \\
\hline Otimas & 3 & 5 & 2 & 53 & & & 2 & 7 & 7 & 171 & 1 & 35 & 2 & 95 & 1 & 41 & 3 & 142 & 21 & 7 \\
\hline Boas & 24 & 40 & 18 & 47 & 13 & 764 & 14 & 50, & 19 & 464 & 11 & 379 & 9 & 428 & 12 & 50,0 & 11 & 523 & 131 & 42 \\
\hline hegres & 14 & 24 & 6 & $15 \%$ & 3 & 176 & 1 & 250 & 11 & 268 & 11 & $37 \%$ & 6 & 285 & 6 & 250 & 6 & 28,5 & 70 & 25 \\
\hline Burin & 1 & $1 \%$ & 管 & 5 & & & & & 1 & 24 & 2 & 69 & & & 1 & 4.1 & & & 6 & 2 \\
\hline$P \operatorname{ssina}$ & 3 & 3,5 & & & & & & & & & 4 & 138 & & & & & & & 3 & 10 \\
\hline $\mathrm{NaOSe}$ & 3 & 35 & 10 & 264 & 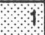 & 60 & s & 179 & 3 & 13 & & & 4 & 192 & 4 & 168 & 1 & 50 & 34 & 12 \\
\hline $\mathrm{SR}$ & 12 & 201 & & & & & & & & & & & & & & & & & 14 & 50 \\
\hline 10tal & 58 & 100 & 38 & 100 & 17 & 100 & 28 & 100 & 41 & 100 & 29 & 100 & 21 & 100 & 24 & 100 & 21 & 100 & 27 & 100 \\
\hline
\end{tabular}

SR. Sem referência

Os dados da Tabela em epigrafe revelam que $131(47,2 \%)$ dos alunos da pesquisa qualificam a expectativa sobre oportunidade de emprego "boa," enquanto que $70(25,2 \%)$ consideram as oportunidades de emprego "regular". Entretanto $34(12,2 \%)$ referem que não conhecem a oportunidade de emprego no mercado de trabalho. 
Tabela 11. Opiniões dos alunos de Enfermagem como pensam em exercer a profissão no ano seguinte à formatura. 1993

\begin{tabular}{|c|c|c|c|c|c|c|c|c|c|c|c|c|c|c|c|c|c|c|c|c|}
\hline \multirow[t]{2}{*}{ Opincos: } & \multicolumn{2}{|c|}{$10 \mathrm{Sem}$} & \multicolumn{2}{|c|}{$2^{\circ}$ sem } & \multicolumn{2}{|c|}{$3^{6} \mathrm{sem}$} & \multicolumn{2}{|c|}{$4^{\circ}$ Sem } & \multicolumn{2}{|c|}{$5 \mathrm{sem}$} & \multicolumn{2}{|c|}{$6^{\circ} \mathrm{Sem}$} & \multicolumn{2}{|c|}{$78 \mathrm{sm}$} & \multicolumn{2}{|c|}{$8^{\circ} \mathrm{Sem}$} & \multicolumn{2}{|c|}{$96 \mathrm{Sem}$} & \multicolumn{2}{|c|}{ otal } \\
\hline & $\mathrm{No}$ & $\%$ & $\mathrm{NO}^{\circ}$ & $\%$ & 10 & $\%$ & No & $\%$ & $N$ & $\%$ & $\mathrm{NO}$ & $\%$ & $\mathrm{NO}^{\circ}$ & $\%$ & No & $\%$ & $N /$ & 8 & $N$ & $\%$ \\
\hline$S P$ & 12 & 20,6 & 3 & 184 & 3 & 176 & 1 & 3,6 & 5 & 221 & 2 & 6,8 & 3 & 14.2 & 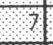 & 291 & 4 & 334 & 4 & 110 \\
\hline $\mathrm{SH}$ & 36 & 623 & 25 & 65 & 12 & 705 & 25 & 89.2 & 26 & 634 & 25 & 86,2 & 17 & 809 & 6 & 666 & 14 & 666 & 96 & To \\
\hline EEn & 2 & 34 & 1 & 2,6 & & & 1 & 36 & 7 & 172 & 1 & 35 & & & & & & & 1 & 43 \\
\hline $\mathrm{NPE}$ & 5 & 86 & 1 & 26 & & & & & & & & & & & & & & & 6 & $2 z$ \\
\hline Outra & 3 & 51 & 4 & 10.7 & 2 & 119 & 1 & 3,6 & 3 & 73 & 1 & 35 & 1 & 49 & & & & & 15 & 54 \\
\hline $\mathrm{SB}$ & H & & & & & & & & 9 & & 1 & & & & 1 & 4.3 & & & 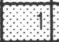 & 04 \\
\hline Total & 58 & 100 & 38 & 100 & 17 & 100 & 28 & 100 & 4 & 100 & 29 & 100 & 21 & 100 & 24 & 100 & 21 & 100 & 27 & 00 \\
\hline
\end{tabular}

Legenda: S.P. Saúde Pública S.H. Serviço hospitalar E.Enf. Escola de Enfermagem N.P.E. Não Pretende exercer, SR. Sem referência.

Percebe-se que $196(70,7 \%)$ dos alunos pensam em exercer a profissão na área hospitalar. Ao analisarmos esta tendência, é provável que ela esteja relacionada ao processo de formação dos alunos de Enfermagem, que ainda passam por um modelo de ensino clínico, caracterizado pelo fortalecimento da atenção médica e hospitalar.

Tabela 12. Alunos que trabalhavam ou não antes de ingressar na Faculdade - 1993

\begin{tabular}{|c|c|c|c|c|c|c|c|c|c|c|c|c|c|c|c|c|c|c|c|c|}
\hline rabaltho & \multicolumn{2}{|c|}{$10 \mathrm{sem}$} & \multicolumn{2}{|c|}{$z^{0} \mathrm{sem}$} & \multicolumn{2}{|c|}{3 Sen } & \multicolumn{2}{|c|}{$4 . \mathrm{sem}$} & \multicolumn{2}{|c|}{$5^{\circ} \mathrm{Sem}$} & \multicolumn{2}{|c|}{$60 \mathrm{sen}$} & \multicolumn{2}{|c|}{$7^{\circ} 5 \mathrm{em}$} & \multicolumn{2}{|c|}{$8^{\circ} \mathrm{sen}$} & \multicolumn{2}{|c|}{$9.5 \mathrm{em}$} & \multicolumn{2}{|l|}{ Total } \\
\hline Remun & $\mathrm{N} \mathrm{N}^{\circ}$ & $\%$ & $\mathrm{~N}^{\mathrm{e}}$ & $\%$ & 10 & $\%$ & $\mathrm{~N}^{\circ}$ & $\%$ & 10 & $\%$ & $\mathrm{~N}^{\mathrm{p}}$ & $\%$ & $\mathrm{No}$ & $\%$ & $\mathrm{~N}$ & $\%$ & $\mathrm{~N}^{\circ}$ & $\%$ & $\mathrm{~N}^{\mathrm{6}}$ & $\%$ \\
\hline in & 3 & 52 & 8 & 210 & 3 & 177 & 17 & 250 & 11 & 26,8 & 8 & 26,6 & a & 286 & 191 & 45.8 & 8 & 23.8 & 62 & 1224 \\
\hline 20 & 55 & 948 & 30 & 19,0 & 14 & 82,3 & 21 & $75 ; 0$ & 80 & 732 & 21 & 724 & 15 & 714 & 13 & 542 & 16 & 76.2 & 215 & 78 \\
\hline 012 & 158 & 100 & 38 & 100 & 17 & 100 & 28 & 100 & 41 & 100 & 29 & 100 & 21 & 100 & 24 & 100 & 21 & 100 & 27 & 100 \\
\hline
\end{tabular}

Observa-se, na Tabela 12, que 215 (77,6\%) dos alunos de Enfermagem, que estão cursando disciplinas, não têm trabalho remunerado, enquanto que $62(22,4 \%)$ referem ter trabalho remunerado. Isso nos aponta que ainda existe um elevado percentual de alunos que vivem sob a tutela dos pais ou parentes.

Tabela 13. Distribuição dos alunos que contribuem ou não com as despesas da família. 1993

\begin{tabular}{|c|c|c|c|c|c|c|c|c|c|c|c|c|c|c|c|c|c|c|c|c|}
\hline \multirow{2}{*}{ Contribuifá } & \multicolumn{2}{|c|}{$1 \mathrm{Sem}$} & \multicolumn{2}{|c|}{$2^{0} \mathrm{Sem}$} & \multicolumn{2}{|c|}{$3^{\circ} \mathrm{Sem}$} & \multicolumn{2}{|c|}{$4 \mathrm{Sem}$} & \multicolumn{2}{|c|}{$5^{6} \mathrm{sem}$} & \multicolumn{2}{|c|}{$6^{\circ} \mathrm{Sem}$} & \multicolumn{2}{|c|}{$8 \mathrm{Sem}$} & \multicolumn{2}{|c|}{$80 \mathrm{Sem}$} & \multicolumn{2}{|c|}{$9^{6} \mathrm{Sem}$} & \multicolumn{2}{|c|}{ Total } \\
\hline & Ne & \% & NO & $\%$ & $N^{\circ}$ & $\%$ & $N^{\circ}$ & $\%$ & $\mathrm{~N}^{0}$ & $\%$ & $N$ & $\%$ & $N$ & $\%$ & $\mathrm{~N}^{\circ}$ & $\%$ & $\mathrm{~N}^{\circ}$ & $\%$ & 18 & $\%$ \\
\hline Controuo nas & & & & & & & & & & & & & & & & & & & & \\
\hline ná sou unico & 1 & 管 & 8 & 185 & 1 & 39 & 3 & 10,8 & 8 & 195 & 5 & 118 & 3 & 14,3 & 9 & 376 & 3 & 14.4 & 40 & 144 \\
\hline Responsaved & & & & & & & & & & & & & & & & & & & & \\
\hline SOU HAHCO & & & & & & & & & & & & & & & & & & & & \\
\hline Responsave & 1 & 17 & 1 & 26 & 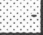 & & 1 & 3.5 & & & & & 1 & 48 & 1 & 4.1 & 1 & 41 & 66 & 22 \\
\hline Náo Contripuo & 56 & 96,6 & 30 & 789 & 36 & 94 & 24 & 85 & 33 & 805 & 24 & 822 & 11 & 809 & 14 & 583 & 19 & 809 & 231 & 834 \\
\hline Jotalo & 58 & 100 & 38 & 100 & 17 & 100 & 28 & 100 & 41 & 800 & 29 & 100 & 81 & 100 & 24 & 100 & 21 & 100 & 277 & 100 \\
\hline
\end{tabular}

Denota-se dos dados da Tabela 13 que $83,4 \%$ (231) não contribuem com as despesas da família, enquanto $14,4 \%$ (40) contribuem com as despesas da 
família, mas não é o único responsável pelo sustento. Isso nos leva a pensar que, de um modo geral, os estudantes de enfermagem têm uma condição sócio-econômica equilibrada não necessitando colaborar no orçamento da familia.

Tabela 14. Distribuição dos alunos que recebem por mês algum auxílio financeiro. 1993

\begin{tabular}{|c|c|c|c|c|c|c|c|c|c|c|c|c|c|c|c|c|c|c|c|c|}
\hline Auxl 0 & \multicolumn{2}{|c|}{$0 \mathrm{sen}$} & \multicolumn{2}{|c|}{$20 \mathrm{sen}$} & \multicolumn{2}{|c|}{$3 \mathrm{sen}$} & \multicolumn{2}{|c|}{$4 \mathrm{Sem}$} & \multicolumn{2}{|c|}{$5 \mathrm{sen}$} & \multicolumn{2}{|c|}{$6 \mathrm{sem}$} & \multicolumn{2}{|c|}{$6 \mathrm{sem}$} & \multicolumn{2}{|c|}{$8 \mathrm{sem}$} & \multicolumn{2}{|c|}{$90 \mathrm{Sem}$} & \multicolumn{2}{|c|}{8012} \\
\hline foratcero & No & 6 & re & o & 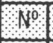 & 6 & 10 & \% & No & \% & Nol & \% & Nol & 6 & No & 6 & N⿴囗十 & 6 & 16 & $\%$ \\
\hline Saláro & 2 & 34 & 2 & 3 & 2 & 11 & 3 & 107 & 4 & 9 & II & 24 & 6 & 286 & 3 & 290 & 1 & 4 & 34 & 123 \\
\hline Mesada & 4 & 76 & 20 & 526 & 10 & 59 & 19 & 678 & 23 & 56.1 & 8 & 49 & 11 & 524 & 12 & 500 & 11 & 524 & 164 & 592 \\
\hline Comissäo & & & 2 & 5 & & & & & & & & & & & & & & & 2 & 0 \\
\hline 6) & & & 3 & की & 勧 & 59 & 4 & 11 & 1 & 2 & 2 & 69 & & & 2 & 88 & 3 & 143 & 13 & 4 \\
\hline BI & 8 & & 勧 & 5 & & & & & 算 & 24 & & & & & 3 & 125 & & & 6 & 22 \\
\hline 0460 & 1 & 12 & 3 & 79 & 3 & 176 & 1 & 5 & 2 & 46 & 8 & 12 & 3 & 95 & & & 1 & 4 & 18 & 64 \\
\hline $\mathrm{SH}$ & 10 & 17 & 6 & 58 & 1 & 5 & 4 & 14 & 10 & 24,6 & 2 & 69 & 2 & 95 & & & 3 & 39 & 40 & 145 \\
\hline ota & 58 & 100 & 38 & 000 & 17 & 100 & 28 & 100 & 41 & 100 & 29 & 100 & 23 & 100 & 24 & 100 & 21 & 100 & 27 & 100 \\
\hline
\end{tabular}

B.T. Bolsa de Trabalho B.E. Bolsa de Estudo SR. Sem referência

Os dados revelam que $164(59,2 \%)$ dos alunos amostrados na pesquisa recebem mesada dos pais; apenas $12,3 \%$ (34) recebem salário proveniente de seu trabalho; $4,7 \%$ (13) têm bolsa de trabalho e 6,5\% (18) recebem de outras fontes que não especificaram. Percebe-se que, na Tabela anterior, $14,4 \%$ (40) contribuem com as despesas da família; no entanto, pode-se evidenciar nesta Tabela que somente 12,3\% (40) recebem salário. Isso nos deixa com o seguinte questionamento: será que os alunos que recebem bolsa de trabalho e/ ou bolsa de estudo contribuem com a despesa familiar?

Tabela 15. Trabalho remunerado exercido pelo estudante antes de ingressar na Universidade. 1993

\begin{tabular}{|c|c|c|c|c|c|c|c|c|c|c|c|c|c|c|c|c|c|c|c|c|}
\hline \multirow{2}{*}{ sominera } & \multicolumn{2}{|c|}{10 sen } & \multicolumn{2}{|c|}{$8 \mathrm{sem}$} & \multicolumn{2}{|c|}{$36 \mathrm{sem}$} & \multicolumn{2}{|c|}{$4 \% \mathrm{en}$} & \multicolumn{2}{|c|}{5 sen } & \multicolumn{2}{|c|}{$6^{\circ} \mathrm{sen}$} & \multicolumn{2}{|c|}{$70 \mathrm{sem}$} & \multicolumn{2}{|c|}{$80 \mathrm{Sem}$} & \multicolumn{2}{|c|}{$9 \mathrm{sen}$} & \multicolumn{2}{|c|}{ Tota } \\
\hline & 10 & 8 & 16 & 6 & 19 & $\%$ & 80 & $\%$ & 10 & $\%$ & $N^{\circ}$ & $\%$ & $\mathrm{NO}$ & $\%$ & $\mathrm{No}$ & $\%$ & 10 & $\%$ & $1^{6}$ & $\%$ \\
\hline Naforaba & 35 & 948 & 3 & 868 & 13 & 764 & 8 & 857 & 33 & 805 & 24 & 82 & 18 & 809 & 17 & 708 & 18 & 85 & 234 & 84 \\
\hline robal/ha & $z$ & 34 & 5 & 13 & 4 & 236 & 9 & 143 & 8 & 195 & 5 & 17 & 4 & 191 & 6 & 250 & 3 & 143 & 4 & 148 \\
\hline 解 & 1 & 18 & & & 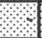 & & & & 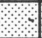 & & & & & & 1 & 48 & & & 8 & $0 \%$ \\
\hline ota & 38 & 100 & 38 & 100 & 11 & 100 & 28 & 100 & 41 & 100 & 29 & 100 & 2 & 100 & 24 & 100 & 21 & 100 & 27 & 100 \\
\hline
\end{tabular}

Observa-se, na Tabela 15, que $84,5 \%$ (234) dos estudantes não exerciam atividades remunerada, representando a grande maioria, enquanto que 14,8 (41) exerciam atividade remunerada que poderá ou não ser conciliada com o Curso que ocupa o estudante em tempo integral. 
Tabela 16. Alunos que concluíram o $2^{\circ} \mathrm{Grau}$, por semestre, e estão cursando enfermagem. 1993

\begin{tabular}{|c|c|c|c|c|c|c|c|c|c|c|c|c|c|c|c|c|c|c|c|c|}
\hline \multirow[t]{2}{*}{ Qurso } & \multicolumn{2}{|c|}{ 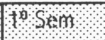 } & \multicolumn{2}{|c|}{$2 . \mathrm{sen}$} & \multicolumn{2}{|c|}{$3^{6} \mathrm{sen}$} & \multicolumn{2}{|c|}{$4 \mathrm{Setn}$} & \multicolumn{2}{|c|}{$58 \mathrm{em}$} & \multicolumn{2}{|c|}{$60 \mathrm{sen}$} & \multicolumn{2}{|c|}{$78 \mathrm{er}$} & \multicolumn{2}{|c|}{$8^{6} S \in \mathrm{m}$} & \multicolumn{2}{|c|}{$\mathrm{Q}^{\mathrm{S}} \mathrm{A}$} & \multicolumn{2}{|c|}{ 1otal } \\
\hline & $\mathrm{A}^{\mathrm{d}}$ & $1 \%$ & 10 & $\%$ & No & $\%$ & $\mathrm{~N}^{\circ}$ & $\%$ & $\mathrm{~N}^{\circ}$ & $\%$ & $\mathrm{~N}^{\circ}$ & 8 & $\mathrm{No}$ & $\%$ & $\mathrm{No}$ & 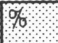 & $\mathrm{N}^{0}$ & $\%$ & $4^{6}$ & $\%$ \\
\hline Cole gra & 52 & 89 & 35 & 92 & 14 & 824 & 25 & 893 & 35 & 85 & 28 & 965 & 13 & 619 & 17 & 70,8 & 13 & 619 & 23 & 83 \\
\hline $\begin{array}{l}\text { Magis } \\
\text { terfo }\end{array}$ & 1 & 10 & 1 & 26 & & & 1 & 36 & 1 & 25 & 8 & 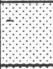 & 1 & 47 & 1 & 42 & & & 6 & 22 \\
\hline $1<01 c 0$ & & 1 & 2 & 5,3 & 3 & 116 & 2 & 7 & 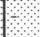 & & & & 6 & 28 & 6 & 250 & 4 & 19 & 23 & 8,3 \\
\hline Qutros & 5 & 86 & 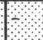 & & 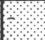 & & & 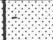 & 7 & 12 & 1 & 35 & 1 & 47 & 8 & & 2 & 95 & 14 & 13 \\
\hline$S \mathrm{~B}$ & 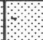 & 3 & 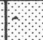 & 1 & \% & 18 & & 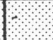 & 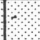 & 6 & 1 & 1 & 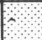 & 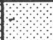 & 1 & 8 & 2 & 95 & 2 & 101 \\
\hline Total & 58 & 100 & 38 & 100 & 17 & 100 & 28 & 100 & 41 & 100 & 29 & 100 & 21 & 100 & 24 & 100 & 8 & 100 & 27 & 100 \\
\hline
\end{tabular}

Legenda: SR- Sem resposta

$\mathrm{Na}$ Tabela acima, percebe-se que $83,7 \%$ (232) alunos concluiram o Colegial, ou seja, o atual ensino do $2^{\circ}$ Grau. $O$ curso técnico ou profissionalizante foi representado por $8,3 \%$ (23) dos estudantes. Denota-se que alguns dos estudantes procuram o curso profissionalizante, pois thes dá condições de trabalhar antes de ingressar em uma Universidade, embora se constate que a maioria procure fazer o Colegial. Conforme tabelas anteriores, esses alunos estão em sua maioria sob a tutela de seus pais.

Tabela 17. Alunos, por semestre, que ingressaram na Universidade logo após ter concluido o $2^{\circ} \mathrm{Grau}$.

\begin{tabular}{|c|c|c|c|c|c|c|c|c|c|c|c|c|c|c|c|c|c|c|c|c|}
\hline \multirow[t]{2}{*}{ Grso } & \multicolumn{2}{|c|}{1 Sen } & \multicolumn{2}{|c|}{$2 \mathrm{sen}$} & \multicolumn{2}{|c|}{$3 \mathrm{sem}$} & \multicolumn{2}{|c|}{$4 \mathrm{sem}$} & \multicolumn{2}{|c|}{$5^{\circ} \mathrm{em}$} & \multicolumn{2}{|c|}{$60 \mathrm{sem}$} & \multicolumn{2}{|c|}{$10 \mathrm{sen}$} & \multicolumn{2}{|c|}{$8 \mathrm{Sem}$} & \multicolumn{2}{|c|}{$9 \mathrm{sen}$} & \multicolumn{2}{|c|}{ Total } \\
\hline & 10 & 8 & 19 & $\%$ & $\mathrm{~N}^{\circ}$ & 6 & $\sqrt{16}$ & $\%$ & 10 & $\%$ & $\mathrm{No}$ & $\%$ & $N^{\circ}$ & $\%$ & $\mathrm{~N}$ & $\%$ & 10 & $\%$ & 16 & $\%$ \\
\hline $\begin{array}{l}\text { A Gonder } \\
01 \text { Grau }\end{array}$ & 36 & 620 & 20 & 52,8 & 9 & 530 & 20 & 74, & 31 & 35 & 19 & 655 & 13 & 619 & 3 & 34 & 13 & 619 & m & 628 \\
\hline Ano & 17 & 293 & 14 & 36,8 & 6 & 35,4 & 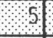 & 119 & 8 & 196 & 10 & 845 & 1 & 4,8 & 3 & 208 & 6 & 28,6 & 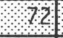 & 260 \\
\hline $\mathrm{Com} 2 \mathrm{Aros}$ & 2 & 3.5 & & & 1 & 58 & (7) & & 1 & 24 & & & 6 & 285 & & & & & 10 & 86 \\
\hline 2 Anos & 3 & 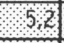 & 3 & 19 & 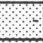 & & 2 & 1 & 1 & 24 & & & 1 & 48 & 4 & 166 & 2 & 95 & 1 & 6 \\
\hline $\mathrm{Sh}$ & 3 & & 1 & 26 & 1 & 58 & 1 & 36 & 1 & & 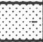 & & 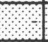 & 管 & 2 & 84 & $m$ & & 4 & 15 \\
\hline Oota & 58 & 100 & 38 & 100 & 17 & 100 & 88 & 100 & 41 & 100 & 29 & 100 & 21 & 100 & 24 & 100 & 21 & 100 & 27 & 100 \\
\hline
\end{tabular}

Legenda: I.A. Imediatamente após.

Constata-se, na Tabela 17, que $174(62,8 \%)$ estudantes do total de 277 , ingressaram na Faculdade logo após o término do $2^{\circ}$ Grau, enquanto 72 $(26,0 \%)$ ingressaram após um ano de concluir o $2^{\circ}$ Grau. Desta forma, acredita-se que um grande número de alunos provavelmente acumulou conhecimentos durante o período de estudo no $1^{\circ}$ e $2^{\circ}$ Graus, ingressando imediatamente no curso almejado. 
Tabela 18. Grau de Instrução da mãe dos alunos de enfermagem no ano de 1993

\begin{tabular}{|c|c|c|c|c|c|c|c|c|c|c|c|c|c|c|c|c|c|c|c|c|}
\hline \multirow[t]{2}{*}{ Gral de Instrusad } & \multicolumn{2}{|c|}{$10 \mathrm{sem}$} & \multicolumn{2}{|c|}{$28 \mathrm{em}$} & \multicolumn{2}{|c|}{3 sen } & \multicolumn{2}{|c|}{$40 \mathrm{sem}$} & \multicolumn{2}{|c|}{$50 \mathrm{sem}$} & \multicolumn{2}{|c|}{$6.5 \mathrm{em}$} & \multicolumn{2}{|c|}{$70 \mathrm{sem}$} & \multicolumn{2}{|c|}{$8 \mathrm{sem}$} & \multicolumn{2}{|c|}{$96 \mathrm{sen}$} & \multirow{2}{*}{ No } & \multirow{2}{*}{ tal } \\
\hline & N1) & 6 & A' & of & $\mathrm{N}^{\circ}$ & \% & $\mathrm{N}^{\circ}$ & $\%$ & No & $\%$ & $\mathrm{NO}$ & $\%$ & $\mathrm{NO}$ & $\%$ & $\mathrm{NO}$ & $\%$ & $\mathrm{~N}^{\circ}$ & $\%$ & & \\
\hline Analfabeta & & & & & & & 1 & 35 & 1 & 24 & 1 & 35 & 1 & 47 & & & & & 4 & 14 \\
\hline Alfabészada & 4 & 6,8 & 3 & 78 & 1 & 58 & 2 & 71 & 1 & 2,4 & 6 & 207 & 2 & 9,6 & 1 & 42 & 3 & 14,4 & 23 & 8.4 \\
\hline Promplo & 4 & 68 & 2 & 52 & 4 & 235 & $z$ & 21 & 2 & 48 & 1 & 35 & 5 & 23,8 & 5 & 20.8 & 1 & 4 & 26 & 94 \\
\hline TG Gratl Conpleto & 4 & 68 & 2 & 32 & 2 & 111 & 5 & 13 & 6 & 146 & 3 & 103 & 3 & 14.3 & 4 & 16 & & & 26 & 94 \\
\hline pleto Gros & 5 & 86 & 1 & 26 & 2 & 117 & 3 & 10,7 & 5 & 12,6 & 6 & 207 & & & 3 & 125 & 1 & 47 & 23 & 84 \\
\hline 2 Grau Conpleto & 18 & 316 & 21 & 55,6 & 5 & 298 & 8 & 28.5 & 15 & 36.5 & 6 & 201 & 6 & 28,6 & 7 & 291 & 10 & 471 & 96 & 34,6 \\
\hline $\begin{array}{l}2^{\circ} \\
\text { Incompleto }\end{array}$ & 11 & 17 & 2 & 52 & 1 & 58 & 1 & 3.5 & 3 & 73 & 3 & 10,3 & 2 & 9,6 & 8 & & 1 & 47 & 1 & 61 \\
\hline $\begin{array}{l}\text { Superior } \\
\text { Completo }\end{array}$ & 16 & 275 & 6 & 158 & 2 & 157 & 6 & 265 & 3 & 2,3 & 2 & 6.8 & 1 & 4.7 & 3 & 12.5 & 4 & 19,1 & 44 & 158 \\
\hline Superior & 4 & 6.8 & & & & & & & 2 & 4.8 & & & 1 & 4,7 & $t$ & 4.2 & & & 10 & 36 \\
\hline $\mathrm{SR}$ & 2 & 34 & 1 & 2.6 & 8 & & & & 3 & 73 & 1 & 35 & 3 & & 8 & 3 & 1 & 4.7 & 8 & 29 \\
\hline Notal & 581 & 100 & 38 & 100 & 17 & 100 & 28 & 100 & 41 & 100 & 29 & 1001 & 21 & 100 & 24 & 100 & 21 & 100 & 27 & 100 \\
\hline
\end{tabular}

Observa-se, na Tabela 18, que $96(34,6 \%)$ das mães dos alunos amostrados concluiram o $2^{\circ}$ Grau, enquanto $44(15,8 \%$ ) das mães têm curso superior completo. Percebe-se que uma grande percentagem dos estudantes já têm uma história anterior de curso universitário, portanto, isso poderá expressar um incentivo aos estudantes para a ascensão profissional.

Tendo em vista o objetivo do estudo caracterizar o perfil do estudante de enfermagem da Universidade Estadual do Ceará, neste estudo procuramos destacar alguns aspectos que remetem à discussão dos resultados.

Sintetizando os aspectos mencionados na pesquisa, pode-se apontar os seguintes:

Os alunos são, em sua maioria, do sexo feminino (88,8\%). Isso não é um dado novo, pois desde o surgimento da Enfermagem no Brasil a categoria que mais predominava na área de Enfermagem era a do sexo feminino. Historicamente, a mulher era considerada desvalorizada, pois não tinha direito a voto, era considerada doméstica, enquanto que a força de trabalho do homem era um marco importante para a guerra e para aumentar os meios de produção. Nesse sentido, a mulher, sendo considerada frágil e desvalorizada, era aproveitada em serviços elementares que não representassem "status" social.

A faixa etária está entre alunos com menos de 20 anos de idade, $28,8 \%$ (80), faixa essa ainda considerada adolescência, momento de conflitos de 
identidade, mudanças de papéis, auto-afirmação, e é nesse momento que o aluno faz a escolha para a profissão que muitas vezes não traduz o que ele realmente deseja. Isso foi confirmado quando perguntamos ao aluno: por que você optou por fazer Enfermagem? Um grande percentual deixou de responder e outros responderam que era por motivos econômicos. 177 (63,9\%) estavam na faixa etária de 20 a 29 anos (Tabela 2 ).

Evidencia-se que 167 (60,3\%) dos estudantes vivem sob a tutela dos pais, enquanto $12(4,3 \%)$ vivem em república ou sozinhos. Isso nos leva a pensar que a emancipação da mulher ainda está por acontecer, pois as alunas dessa amostra, em sua grande maioria, vivem sob a tutela dos pais ou parentes, indicando assim a persistência de traços culturais paternalistas, de proteção absoluta aos filhos, tirando-lhes a iniciativa de se auto-sustentarem.

Os dados da Tabela 5 mostram que $245(88,4 \%)$ dos alunos vivem em casa própria, o que denota que esses alunos são de situação econômica favorecida, pois a maioria do povo brasileiro não tem condições de ter uma casa própria, devido às desigualdades sociais que afeta a população de baixa renda.

Os dados da Tabela 6 revelam que 129 (46.5\%) dos alunos estudaram em cursinho para ingressar na Enfermagem. Isso leva a uma reflexão: será que a escola brasileira de $1^{\circ}$ e $2^{\circ}$ graus está preparando, adequadamente, o aluno para ingressar no mundo universitário? Essa é uma questão que todos nós devemos refletir. Será que a universidade também está cumprindo o seu papel de educador e formador de senso crítico desses alunos, que estão vivenciando o processo de educar e pesquisar para aprender? 134 (48,3\%) dos alunos não frequentaram cursinho. Existe um equilibrio entre os que ingressaram na universidade tendo frequentado cursinho e os que não frequentaram.

Observa-se, à luz dos dados da Tabela 8, que 260 (93,8\%) dos alunos amostrados prestaram vestibular para outros cursos em outras instituições, dai a preocupação se esses alunos estavam seguros de sua opção pela Enfermagem, ou essa escolha se deu só para ter a oportunidade de ingressar na Universidade, pois ser universitário representa um "status".

Percebe-se uma certa contradição nas respostas, quando esses alunos colocam que escolheram a profissão por inclinação 191 (69,0\%), mas na Tabela 8 revelaram que realizaram vestibular para outros cursos. Dos alunos que compartilharam da pesquisa, $53(19,1 \%)$ deixaram sem resposta a questão e somente uma pequena percentagem optou pela profissão, fazendo o teste vocacional, $5,4 \%(15)$.

Os dados da Tabela 11 mostram que os alunos pensam exercer a profissão na área hospitalar. Essa tendência já era de se esperar, pois o paradigma ainda vigente nas escolas de enfermagem brasileiras ainda é o modelo hospitalocêntrico, voltado para os aspectos biológicos, esquecendo-se 
de que o ser humano é multidimensional, portanto é um ser que transcende os aspectos clínicos, tendo que ser compreendido como um indivíduo que integra uma sociedade cheia de conflitos e contradições. Esse desvelar do ser humano como parte integrante da sociedade é que precisa ser encarado pelos que fazem a saúde, principalmente no sistema educacional que tem forte influência no pensar e fazer. Portanto, essa tendência pela área hospitalar não acontece por acaso na UECE, mas passa pela concepção dos docentes de como ele encara a realidade, pelas políticas sociais e por determinantes estruturais. Esse paradigma poderá ser superado se houver vontade politica e um esforço coletivo.

Os dados da Tabela 12 denotam que 215 (77,6\%) dos alunos não têm trabalho remunerado, o que vem fortalecer a idéia da proteção dos pais, como um forte fator cultural em nossa sociedade.

Dos amostrados na Tabela 13, 231 (83,4\%), não contribuem com a despesa da familia, havendo um pequeno percentual de 46 (16,6\%) que revelaram contribuir na renda familiar.

Das respostas apontadas pelos alunos de Enfermagem, 164 (59,2\%) recebem mesada dos pais. Os dados da Tabela 13, confrontados com os da Tabela 12, evidenciam uma contradição: $215(77,6 \%)$ dizem que não têm trabalho remunerado, no entanto $59,2 \%$ apontam que recebem mesada e $26,3 \%$ recebem outros proventos, como bolsa de pesquisa e de trabalho. Isso nos leva a seguinte reflexão: os alunos bolsistas utilizam o dinheiro da bolsa para despesas familiares ou para recursos que aprofundem seus conhecimentos, tais como livros, entre outros? Observa-se que $234(84,4 \%)$ dos alunos não tinham trabalho remunerado antes de ingressar na Universidade.

Os dados da Tabela 18 revelam que os 200 (72,2\%) alunos que ingressaram no Curso de Enfermagem fizeram vestibular uma vez.

Considera-se que os aspectos anteriormente referidos e já analisados conduzem a levantar hipóteses acerca da relevância deste estudo, sobretudo enquanto contribuição para um debate, seja em relação ao estudante, ao processo educacional ou a propósito de subsidio para uma reflexão a nível curricular. Portanto, sintetizando o estudo como marco fundamental no processo de educar e fazer enfermagem, estudos de perfil de aluno servirão como suporte à estrururação de currículos e planos de ensino e contribuirão com a seleção de critérios de avalição de aprendizagem do aluno. 
ABSTRACT: The study approaches the main characteristics of nursing students concerning demographic aspects, demographic mobility, instruction level, occupational situation, attendance to another University Course, information about career and the oportunity of work among other items. As an instrument to collect data, we have used a questionnaire with open and closed questions. The data analysis was based on the students' answers, having as a direction a critical view on these data, which have been compiled in tables. The research outcome revealed that most nursing students are female $(88 \%)$; $57 \%$ come from Fortaleza - CE and $60.2 \%$ still live under their parents' guardianship. From the investigated students $18.7 \%$ parents have graduated from a University and $88.4 \%$ have lived in rented houses.

KEYWORD: Profile - Nursing Students.

\section{REFERÊNCIAS BIBLIOGRÁFICAS.}

1.BOLL et al. Qual é o perfil do estudante de enfermagem da UFRGS. R. Gaucha de Enferm., Porto Alegre, v.9, n.2, p.118-124, dez. 1988.

2. CALDAS, Marcia Alvarenga de Mello. Pensamentos e experiências na área de saúde de pessoas que vivenciam o adolescer. uma abordagem fenomenológica. São Paulo, 1991. 186p.

3. FERREIRA, Aurélio Buarque de Holanda. Médio dicionário da Língua Portuguesa. Rio de Janeiro: Nova Fronteira, 1985.

4. FERREIRA-SANTOS, Célia Almeida. A Enfermagem como profissão: Estudo num Hospital Escola. São Paulo: Pioneira. 1973.

5. ROLIN, Eni de Jesus; TAVARES, Maria Solange Guarino; FRANCO, Lais Helena R. O. \& OLIVEIRA, Francinete de Lima. Perfil do aluno ingressante nos cursos de ensino Superior de Enfermagem do Estado de São Paulo-1 993. São paulo, 1993. 18p. (mimeografado)

6. MANZOLLI, Maria Cecília \& MONTELEONE, Z. Caracterização do estudante de enfermagem. Enf. Novas Dimens., São Paulo, v.3, n.4. p.206-14, jul./ago., 1977.

7. NAKAMAE, Djair Daniel \& COSTA, Maria Lúcia A. S. Semelhanças e as diferenças de perfil de estudantes de escolas de enfermagem oficiais $e$ particulares da Região de Grande São paulo. São Paulo, 1988. 30p. (mimeografado)

8. NAKAMAE, Djair Daniel \& COSTA, Maria Lúcia A. Mudanças de Perfil do estudante de Enfermagem de São Paulo em quinze anos- 1973 a 1988. São Paulo, 1989,65p. (Mimeografado). 
9. ORGANIZAÇÃO MUNDIAL DE LA SALUD. El embarazo y el aborto de la adolescência. Ginebra, 1975. p.10 (Série de informes técnicos).

10. PASTORE, J. \& PEROSA, G. G. O estudante Universitário em São Paulo. Instituto de Pesquisas da USP, 1971(Mimeografado).

11. PIRES, Denise. Hegemonia Médica na Saúde e a enfermagem: Brasil 1500 a 1930. São Paulo: Cortez, 1989. 155p.

12. SCHMIEDELK, R.A. Adolescent Identity formation and the organizational structure of Highs Schools._Adolecesce. V.4, n. 14, p.191-6, 1979. 